\title{
Neoadjuvant treatment of localized pancreatic adenocarcinoma
}

\author{
Alexis D. Leal^, Wells A. Messersmith, Christopher H. Lieu \\ Division of Medical Oncology, School of Medicine, University of Colorado, Aurora, CO, USA \\ Contributions: (I) Conception and design: AD Leal; (II) Administrative support: AD Leal; (III) Provision of study materials or patients: AD Leal; (IV) \\ Collection and assembly of data: AD Leal; (V) Data analysis and interpretation: AD Leal; (VI) Manuscript writing: All authors; (VII) Final approval \\ of manuscript: All authors. \\ Correspondence to: Alexis D. Leal, MD. Mail Stop 8117, Research One South, 12801 E 17th Avenue, Rm 8123, Aurora, CO 80045, USA. \\ Email: Alexis.Leal@cuanschutz.edu.
}

\begin{abstract}
Pancreatic adenocarcinoma is one of the deadliest malignancies worldwide and its incidence is rising in the United States. The only potential curative treatment for this disease is surgical resection, however, due to the lack of an effective screening strategy, the majority of patients present with advanced or metastatic disease which preclude resectability. Further, the best clinical outcomes occur in those patients that receive multimodality treatment with surgical resection combined with chemotherapy with or without the addition of radiation therapy. Despite decades of innovation in treatment modalities, including chemotherapy, radiation therapy and advancements in surgical techniques, the long term outcomes for this disease remain poor with high rates of both local and distant recurrence despite curative intent treatment. The dismal outcomes of this disease highlight the dire need for more effective treatment strategies and therapeutics. This review focuses on the treatment of localized pancreatic adenocarcinoma with an in depth review of the literature to support the use of chemotherapy in the adjuvant and neoadjuvant setting in this disease and exploration and discussion of the growing paradigm shift to neoadjuvant treatment. Further, this review highlights the ongoing and planned clinical trials evaluating neoadjuvant treatment strategies and novel therapeutics in localized pancreatic adenocarcinoma.
\end{abstract}

Keywords: Pancreas adenocarcinoma; neoadjuvant; chemotherapy; locally advanced; borderline resectable

Submitted Jun 30, 2020. Accepted for publication Oct 07, 2020.

doi: 10.21037/jgo-20-250

View this article at: http://dx.doi.org/10.21037/jgo-20-250

\section{Introduction}

Pancreatic adenocarcinoma is the $4^{\text {th }}$ leading cause of cancer death among men and women in the United States (U.S.) (1). Further, the incidence of pancreas cancer is rising in the U.S., which is thought to be related to the increasing prevalence of obesity among the population and longer life expectancy, amongst other unknown factors. Despite many advances in the treatment of pancreas adenocarcinoma, prognosis remains poor with an overall 5-year relative survival rate of $9 \%$ (1). Surgical resection is the only potential curative treatment for pancreatic adenocarcinoma.
However, due to the lack of early symptoms and no current recommended screening strategy, up to $80 \%$ of patients present with advanced or metastatic disease at time of diagnosis precluding resectability (2).

The National Comprehensive Cancer Network (NCCN) defines resectability at time of diagnosis to one of three categories, resectable, borderline resectable or locally advanced (3).

(I) Surgically resectable is defined as lacking arterial tumor contact with the celiac axis (CA), superior mesenteric artery (SMA), or common hepatic artery (CHA), and no tumor contact with the

^ ORCID: 0000-0002-0652-8151. 
superior mesenteric vein (SMV) or portal vein (PV) or $\leq 180$ degrees of contact without vein contour irregularity and without evidence of distant organ or lymph node metastasis.

(II) Borderline resectable is defined as solid tumor contact with the CHA without extension to the CA or hepatic artery bifurcation, allowing for safe and complete resection and reconstruction; solid tumor contact with the SMA $\leq 180$ degrees; solid tumor contact with the $\mathrm{CA} \leq 180$ degrees or contact $>180$ degrees without involvement of the aorta and intact and uninvolved gastroduodenal artery; solid tumor contact with the SMV or PV $>180$ degrees or contact of $\leq 180$ degrees with contour irregularity of the vein or thrombosis of the vein with suitable vessel proximal and distal to the site of involvement allowing for safe and complete resection and vein reconstruction; solid tumor contact with the inferior vena cava. No evidence of distant organ or lymph node metastasis.

(III) Locally advanced is defined as solid tumor contact with either the SMA or CA $>180$ degrees or solid tumor contact with the CA and aortic involvement or unreconstructable SMV or PV due to tumor involvement or occlusion. No evidence of distant organ or lymph node metastasis.

Despite curative intent surgery, survival rates following surgery alone remain poor with median overall survival (mOS) ranging from 11-20 months (4-8). The best outcomes for patients with this disease have been seen in those who have received multimodality therapy with surgery and systemic chemotherapy, with or without radiation therapy.

This review will focus on the chemotherapeutic management of localized pancreatic adenocarcinoma with a review of the historical data to support adjuvant therapy including a review of recent updates from recently completed and reported adjuvant clinical trials, review of the data to support neoadjuvant therapy in localized pancreatic cancer and review of ongoing neoadjuvant clinical trials in this setting.

\section{Adjuvant therapy}

The current standard of care for patients with resectable pancreatic adenocarcinoma is for upfront surgical resection followed by adjuvant chemotherapy. Table 1 reviews the major phase III clinical trials investigating the use of adjuvant therapy for the management of resectable pancreas adenocarcinoma.

\section{Adjuvant pancreas adenocarcinoma clinical trials}

The Gastrointestinal Tumor Study Group (GITSG) conducted a prospective randomized study in 43 patients with resected pancreas adenocarcinoma to determine if adjuvant fluoropyrimidine-based chemoradiation helped to reduce locoregional recurrence (4). This study was terminated early due to low accrual and due to observation of large survival differences between the two treatment groups ( 20 vs. 11 months; $\mathrm{P}=0.035$ ) in favor of the adjuvant therapy group. The GITSG registered an additional 30 patients with similar characteristics to those included in the original study and confirmed a survival benefit with adjuvant chemoradiation compared to observation alone (two year survival $43 \%$ vs. $18 \%$, respectively) (9).

The ESPAC-1 trial was a multicenter trial that utilized a two-by-two factorial design to randomly assign patients with resected pancreatic ductal adenocarcinoma to treatment with either chemoradiotherapy alone (20 Gy in 10 daily fractions over 2 weeks with $500 \mathrm{mg} / \mathrm{m}^{2}$ fluorouracil intravenously on days 1-3, repeated after 2 weeks), chemotherapy alone (fluorouracil $425 \mathrm{mg} / \mathrm{m}^{2}$ and folinic acid $20 \mathrm{mg} / \mathrm{m}^{2}$ daily for 5 days, monthly for 6 months), chemoradiotherapy and chemotherapy or observation alone (5). A total of 289 patients were randomized, with 73 patients assigned to chemoradiotherapy alone, 75 patients to chemotherapy alone, 72 patients to chemoradiotherapy plus chemotherapy and 69 patients to observation. This was the first trial to show a survival benefit with the use of adjuvant fluorouracil, with mOS of 19.7 months (95\% CI, 16.4-22.4 months) for patients assigned to adjuvant chemotherapy compared to 14.0 months (95\% CI, 11.9 16.5) for those assigned to observation alone [hazard ratio (HR) 0.66 ; $95 \%$ CI, 0.52-0.83]. In contrast to the findings of the GITSG study, chemoradiation was found to not only provide no additional benefit to either observation alone or adjuvant chemotherapy, it was actually harmful to patients, with shorter survivals in patients that received chemoradiation compared to those who either received observation alone or adjuvant chemotherapy alone. This trial has been criticized due to the use of substandard radiotherapy technique with split course chemoradiation and the lack of central review and quality assurance of the radiotherapy administered during this trial (17-19).

The CONKO-001 trial was a German, multicenter, 
Table 1 Key randomized phase III clinical trials of adjuvant treatment in pancreas adenocarcinoma

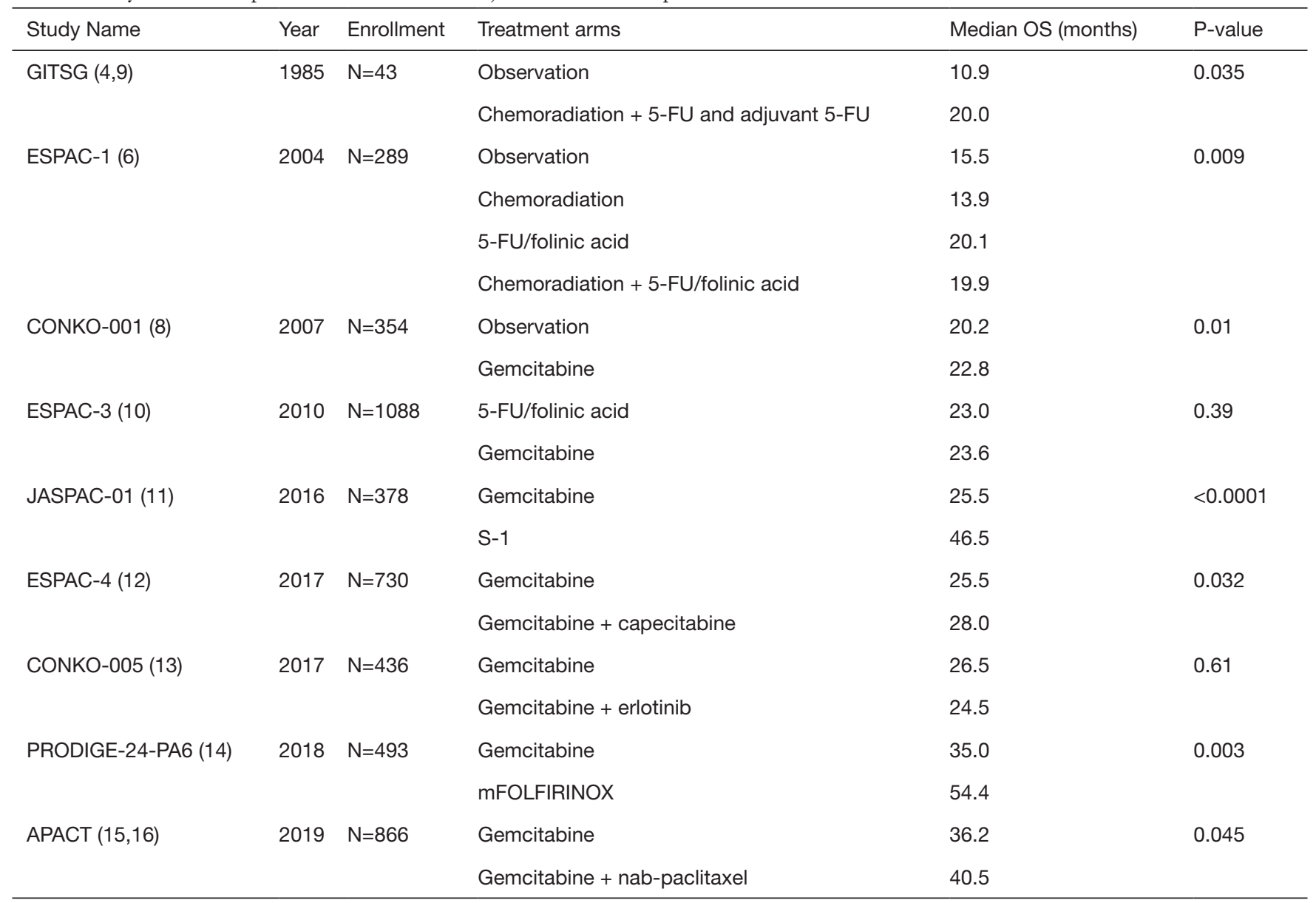

5-FU, 5-fluorouracil; FOLFIRINOX, fluorouracil, leucovorin, irinotecan, oxaliplatin; mFOLFIRINOX, modified FOLFIRINOX; OS, overall survival.

phase III, randomized trial which randomized 354 patients with resected pancreatic adenocarcinoma to either adjuvant therapy with gemcitabine $\left(1,000 \mathrm{mg} / \mathrm{m}^{2}\right.$ days $1,8,15$ every 28 days for 6 months) or observation alone (8). Post-operative tumor markers (CEA/CA 19-9) had to be below 2.5 times the upper limit of normal in order to be eligible. The patients that received adjuvant gemcitabine had statistically significant improvement in survival, with mOS of 22.8 months in the gemcitabine group compared to 20.2 months in the observation group (HR 0.76 ; 95\% CI, $0.61-0.95 ; \mathrm{P}=0.01)$. Five- and ten-year OS was significantly longer in those that received adjuvant gemcitabine with $20.7 \%$ (95\% CI, $14.7-26.6 \%$ ) and $12.2 \%$ (95\% CI, $7.3-$ $17.2 \%$ ) alive at five and ten years respectively, compared to $10.4 \%$ (95\% CI, $5.9-15.0 \%$ ) and $7.7 \%$ (95\% CI, $3.6-11.8 \%)$ respectively, in those that received observation alone. This study was the first to show a survival benefit of gemcitabine in the adjuvant setting.

The ESPAC-3 trial was a multicenter, phase III, randomized trial which randomized 1088 patients with resected pancreatic adenocarcinoma to treatment with either fluorouracil plus folinic acid (folinic acid $20 \mathrm{mg} / \mathrm{m}^{2}$, followed by fluorouracil $425 \mathrm{mg} / \mathrm{m}^{2}$ days $1-5$ every 28 days) or gemcitabine for 6 months (10). There were no restrictions on baseline serum CA 19-9 levels. This study showed similar mOS between the two adjuvant regimens, with $\mathrm{mOS}$ for patients treated with fluorouracil plus folinic acid of 23.0 months (95\% CI, 21.1-26.4 months) compared to 23.6 months (95\% CI, 21.4-26.4 months) for those treated with gemcitabine. Progression free survival (PFS) was also similar between the treatment groups, with median PFS (mPFS) of 14.1 months (95\% CI, 12.5-15.3 months) for fluorouracil plus folinic acid compared to 14.3 months (95\% CI, 13.5-15.6 months) for gemcitabine. There were, 
however, significant differences in toxicity profiles between the two regimens, with $14 \%$ of patients who received fluorouracil plus folinic acid reporting treatment related serious adverse events compared to $7.5 \%$ in those who received gemcitabine $(\mathrm{P}<0.001)$. This study established gemcitabine as the new standard of care adjuvant chemotherapy regimen.

The JASPAC-01 trial was a multicenter, Japanese, phase III, non-inferiority study which randomized 377 patients with resected stage I-III pancreatic adenocarcinoma to either gemcitabine for 6 months or S-1 (dosed orally by body surface area and administered twice daily for 28 days followed by 14 days rest, once every 6 weeks for up to 4 cycles) (11). This study was discontinued early at the interim analysis for efficacy, at which point the HR of mortality was 0.57 (95\% CI, 0.44-0.72; $\mathrm{P}<0.0001$ for both non-inferiority and superiority), associated with a 5 -year OS of $24.4 \%$ in the gemcitabine group, compared to $44.1 \%$ in the S-1 group. This trial established S-1 as a new standard of care option in Japanese patients, however, the generalizability of these data to a Western population remain unknown as only Japanese patients were enrolled.

The ESPAC-4 trial was a multicenter, phase III, randomized trial which randomized 730 patients with resected pancreatic adenocarcinoma to treatment with either six cycles of gemcitabine alone or in combination with oral capecitabine $\left(1,660 \mathrm{mg} / \mathrm{m}^{2}\right.$ administered for 21 days followed by 7 days rest) (12). There were no restrictions on baseline serum CA 19-9 levels. Survival was significantly longer in patients treated with adjuvant gemcitabine plus capecitabine compared to gemcitabine alone, with mOS of 28.0 months (95\% CI, 23.5-31.5 months) compared to 25.5 months (95\% CI, 22.7-27.9 months) respectively (HR 0.82 ; 95\% CI, 0.68-0.98). Further, the overall toxicity of the combination of gemcitabine/capecitabine was not significantly higher than that of gemcitabine alone and side effects were manageable. This study confirmed the survival benefit of gemcitabine from the ESPAC-3 study and established gemcitabine/capecitabine as the new standard of care adjuvant chemotherapy regimen at that time.

The PRODIGE-24-PA6 trial was a multicenter, phase III, randomized trial which randomized 493 patients with resected pancreatic adenocarcinoma to either modified FOLFIRINOX (mFOLFIRINOX) (oxaliplatin $85 \mathrm{mg} / \mathrm{m}^{2}$, irinotecan $150 \mathrm{mg} / \mathrm{m}^{2}$, leucovorin $400 \mathrm{mg} / \mathrm{m}^{2}$ and fluorouracil 2,400 mg/m $\mathrm{m}^{2}$ every 2 weeks) or gemcitabine $\left(1,000 \mathrm{mg} / \mathrm{m}^{2}\right.$ weekly for 3 weeks of every 4 weeks) for 24 weeks (14). This study required patients to have a post-operative serum CA $19-9$ less than $180 \mathrm{U} / \mathrm{mL}$ within 21 days prior to randomization for eligibility. Survival was significantly longer in patients treated with mFOLFIRINOX with mOS 54.4 months compared 35.0 months in those treated with gemcitabine (HR 0.64; 95\% CI, 0.48-0.86; $\mathrm{P}=0.003)$. Median disease free survival (mDFS) was also significantly longer in the mFOLFIRINOX arm (21.6 months) compared to gemcitabine (12.8 months) (HR 0.58; 95\% CI, 0.46-0.73; $\mathrm{P}<0.001)$. The rate of grade 3 or 4 adverse events was higher in the mFOLFIRINOX arm (75.9\%) compared to the gemcitabine arm (52.9\%). This study establishes mFOLFIRINOX as the new standard of care adjuvant treatment regimen for fit patients (ECOG $0-1$ ) and has shown the longest survival thus far (for both treatment arms) in patients with pancreas adenocarcinoma treated in the adjuvant setting.

The APACT trial was a multicenter, phase III randomized trial which randomized 866 patients with resected pancreatic adenocarcinoma to either gemcitabine $\left(1,000 \mathrm{mg} / \mathrm{m}^{2}\right.$ weekly for 3 weeks of every 4 weeks $)$ alone or in combination with nab-paclitaxel $\left(125 \mathrm{mg} / \mathrm{m}^{2}\right.$ weekly for 3 weeks of every 4 weeks) for 24 weeks (15). This study required CA $19-9<100 \mathrm{U} / \mathrm{mL}$ for eligibility. This study failed to meet its primary endpoint of independently assessed DFS with mDFS of 19.4 months in the gemcitabine/nab-paclitaxel arm compared to 18.8 months in the gemcitabine monotherapy arm (HR 0.82; 95\% CI, 0.69-0.97; $\mathrm{P}=0.018)$. Investigator assessed DFS was 16.6 months in the gemcitabine/nab-paclitaxel arm compared to 13.7 months in the gemcitabine monotherapy arm (HR 0.82; 95\% CI, 0.69-0.97; $\mathrm{P}=0.017$ ). Updated OS data from the intention-to-treat population was recently presented and showed longer OS in those patients treated with combination therapy (41.8 months) compared to those treated with monotherapy (37.7 months) (HR 0.81; 95\% CI, 0.68-0.97; $\mathrm{P}=0.047)$, suggesting this is an active regimen in the adjuvant setting (16).

Based upon these data, the current standard of care options for the adjuvant treatment of pancreatic adenocarcinoma include mFOLFIRINOX, gemcitabine/ capecitabine, single agent gemcitabine or fluoropyrimidine based treatment, with selection of treatment based upon patient clinical factors. For a clinically fit patient with ECOG performance status of $0-1$, six months of mFOLFIRINOX is recommended. If a fit patient is not a candidate for mFOLFIRINOX, six months of gemcitabine/ capecitabine is recommended. Consideration of single 
Table 2 Completed randomized phase II or III clinical trials of neoadjuvant treatment in pancreas adenocarcinoma

\begin{tabular}{|c|c|c|c|c|c|c|}
\hline Study name & Year & Enrollment & Setting & Treatment arms & Median OS (months) & $P$ value \\
\hline \multirow{2}{*}{ PACT-15 (28) } & \multirow{2}{*}{2018} & \multirow{2}{*}{$\mathrm{N}=88$} & \multirow{2}{*}{ RPC } & Surgery + adjuvant PEXG & 26.4 & \multirow{2}{*}{ NR } \\
\hline & & & & PEXG $\times 3+$ surgery + adjuvant PEXG $\times 3$ & 38.2 & \\
\hline $\begin{array}{l}\text { Seoul National University } \\
\text { Hospital (29) }\end{array}$ & 2018 & $\mathrm{~N}=50$ & BRPC & $\begin{array}{l}\text { Surgery + adjuvant gemcitabine-based } \\
\text { chemoradiation + adjuvant gemcitabine }\end{array}$ & 12.0 & 0.028 \\
\hline \multirow[t]{2}{*}{ PREP-02/JSAP-05 $(30,31)$} & \multirow[t]{2}{*}{2019} & \multirow[t]{2}{*}{$\mathrm{N}=362$} & \multirow[t]{2}{*}{ RPC } & Surgery + adjuvant S-1 & 26.6 & \multirow[t]{2}{*}{0.015} \\
\hline & & & & Gemcitabine/S-1 + surgery + adjuvant S-1 & 36.7 & \\
\hline PREOPANC-1 (32) & 2020 & $\mathrm{~N}=246$ & RPC, BRPC & Surgery + adjuvant gemcitabine & 14.3 & 0.96 \\
\hline \multirow[t]{2}{*}{ SWOG S1505 (33) } & \multirow[t]{2}{*}{2020} & \multirow[t]{2}{*}{$N=102$} & \multirow[t]{2}{*}{ RPC } & FOLFIRINOX $\times 6+$ surgery + FOLFIRINOX $\times 6$ & 22.4 & \multirow[t]{2}{*}{ NR } \\
\hline & & & & $\begin{array}{l}\text { Gemcitabine/nab-paclitaxel } \times 3+\text { surgery }+ \\
\text { gemcitabine/nab-paclitaxel } \times 3\end{array}$ & 23.6 & \\
\hline
\end{tabular}

BRPC, borderline resectable pancreas cancer; FOLFIRINOX, fluorouracil, leucovorin, irinotecan, oxaliplatin; NR, not reported; OS, overall survival; PEXG, cisplatin, epirubicin, gemcitabine and capecitabine; RPC, resectable pancreas cancer.

agent gemcitabine or fluoropyrimidine based treatment should be reserved for those patients that are less fit and unlikely to tolerate either mFOLFIRINOX or gemcitabine/ capecitabine.

\section{Neoadjuvant therapy}

Neoadjuvant therapy has been increasingly used in the management of resectable and borderline resectable pancreatic cancer, both with and without radiation. This paradigm has been largely adopted by academic institutions, however, the predominance of data to support this strategy is based upon single institution studies (20-22) and metaanalyses (23-26) with few data available from randomized phase II and III clinical trials.

\section{Rationale for neoadjuvant therapy}

Approximately $60 \%$ of patients who undergo upfront surgical resection do not receive adjuvant therapy, with over half of patients not receiving adjuvant treatment due to the development of post-operative complications (27). Further, pancreatic adenocarcinoma is thought to be a systemic disease at diagnosis, with development of metastases as an early event. This is corroborated by the incredibly high rate of local and distant recurrence despite adjuvant treatment.

The potential benefits of neoadjuvant therapy include early treatment of micrometastatic disease, tumor downstaging, increase in $\mathrm{R} 0$ resection rates, improved systemic delivery of treatment with greater completion rates of intended treatment, and the ability to test tumor biology in vivo, which may potentially spare patients that either do not tolerate chemotherapy or progress through chemotherapy from a major surgery that is unlikely to be curative.

\section{Neoadjuvant pancreas adenocarcinoma clinical trials}

Table 2 reviews the completed phase II and III clinical trials investigating the use of neoadjuvant therapy for the management of resectable and borderline resectable pancreas adenocarcinoma.

The PACT-15 trial was a multicenter, phase II/III, randomized clinical trial in Italy which randomized 93 patients with untreated, pathologically confirmed stage I-II resectable pancreatic adenocarcinoma to either surgery followed by adjuvant gemcitabine $\left(1,000 \mathrm{mg} / \mathrm{m}^{2}\right.$ weekly for 3 weeks of every 4 weeks for 6 cycles; Arm A), surgery followed by adjuvant PEXG (cisplatin $30 \mathrm{mg} / \mathrm{m}^{2}$, epirubicin $30 \mathrm{mg} / \mathrm{m}^{2}$, and gemcitabine $800 \mathrm{mg} / \mathrm{m}^{2}$ on days 1 and 15 
every 4 weeks with capecitabine $1,250 \mathrm{mg} / \mathrm{m}^{2}$ on days $1-28$; Arm B) or perioperative PEXG (three cycles neoadjuvant and 3 cycles adjuvant; Arm C) (28). The primary endpoint of this study was event free survival at 1 year in the perprotocol population. Among the 88 patients included in the final study population ( 5 were excluded due to center non-compliance with the protocol), 26 were assigned to Arm A, 30 to Arm B and 32 to Arm C. In the perprotocol population, $23 \%(n=6 / 30)$ of patients in Arm A, $50 \%(n=15 / 30)$ of patients in Arm B, and 66\% ( $n=19 / 29)$ of patients in Arm $\mathrm{C}$ were event free at 1 year. Further evaluation of this regimen in this setting in a larger, phase III trial was not pursued due to the development of other effective chemotherapy regimens, but this study showed evidence of efficacy with a neoadjuvant treatment approach.

A phase II/III, multicenter, randomized controlled trial in Korea randomized 50 patients with borderline resectable pancreatic cancer to either neoadjuvant gemcitabinebased chemoradiation (54 Gy external beam radiation) followed by surgery or immediate surgery followed by adjuvant chemoradiation at high-volume centers (29). The primary endpoint was 2-year survival. In the intention to treat (ITT) analysis, the mOS and 2-year survival were significantly improved in the neoadjuvant chemoradiation arm compared to the immediate surgery arm (21 months, $40.7 \%$ vs. 12 months, 26.1\%; HR 1.495; 95\% CI, 0.663.36; $\mathrm{P}=0.028$ ). The rates of $\mathrm{R} 0$ resection were higher in the neoadjuvant chemoradiation arm $(51.8 \%)$ compared to the immediate surgery arm $(26.1 \%, \mathrm{P}=0.004)$. Rates of completion of intended treatment were higher in the neoadjuvant chemoradiation group with $96 \%(n=26 / 27)$ of patients completing neoadjuvant treatment compared to $72 \%(n=13 / 18)$ of patients that underwent upfront surgical resection. This study was closed early due to the statistical significance of neoadjuvant therapy efficacy.

The PREP-02/JSAP-05 was a randomized, phase II/ III clinical trial in Japan which randomized 364 patients with biopsy proven, resectable, pancreas adenocarcinoma to either 2 cycles of neoadjuvant gemcitabine $\left(1,000 \mathrm{mg} / \mathrm{m}^{2}\right.$ on days 1,8$)$ and S-1 $\left(40 \mathrm{mg} / \mathrm{m}^{2}\right.$ twice daily on days $\left.1-14\right)$ followed by surgical resection and 6 months of adjuvant S-1 or immediate surgery followed by adjuvant S-1 (30). The primary endpoint of the phase III portion of this study was OS. Among the ITT population, the mOS was superior in those treated with neoadjuvant therapy followed by surgical resection and adjuvant therapy (36.7 months) compared to those who underwent upfront resection and adjuvant therapy (26.6 months) (HR 0.71; 95\% CI,
0.55-0.94; $\mathrm{P}=0.015)$. Further, there was a significant decrease in pathologic nodal metastases in those treated with neoadjuvant therapy compared to the upfront resection group $(59.6 \%$ vs. $81.5 \%$; $\mathrm{P}<0.01)$ (31). There were no differences in resection rates, $\mathrm{R} 0$ resection rate or perioperative outcomes between the two groups. Similar to other studies of S-1 in a purely Asian population, it is difficult to extrapolate these data to a Western population. However, these data support the use of a neoadjuvant approach in an Asian population.

The PREOPANC-1 trial was a multicenter, phase III, randomized trial which included patients with resectable or borderline resectable pancreatic adenocarcinoma and randomly assigned patients to receive neoadjuvant chemoradiation (consisting of 3 cycles of gemcitabine $1,000 \mathrm{mg} / \mathrm{m}^{2}$ on days 1 and 8 every 21 days, with the second cycle combined with 15 fractions of 2.4 Gy to the pancreatic tumor and lymph nodes) followed by 4 cycles of adjuvant gemcitabine or to immediate surgical resection followed by 6 cycles of adjuvant gemcitabine (32). The primary endpoint of this study was OS by ITT. Two hundred and forty-six patients were randomized, one hundred and nineteen to pre-operative chemoradiation and one hundred and twenty seven to immediate surgery. This study did not meet its primary endpoint, with mOS of 16.0 months in the preoperative chemoradiation arm compared to 14.3 months in the immediate surgery arm (HR 0.78 ; 95\% CI $0.58-1.05$; $\mathrm{P}=0.096$ ). Survival analysis of the patients that underwent surgical resection and initiated adjuvant chemotherapy favored the pre-operative chemoradiation arm with mOS 35.2 months compared to 19.2 months $(\mathrm{P}=0.029)$. The $\mathrm{R} 0$ resection rate was higher $(71 \%)$ in the pre-operative chemoradiation arm compared to the immediate surgery arm $(40 \%)(\mathrm{P}<0.001)$. Further, patients enrolled in the pre-operative chemoradiation arm had superior DFS, locoregional failure-free interval and lower rates of lymph node involvement, presence of lymphovascular invasion and perineural invasion.

The SWOG S1505 trial was a multicenter, phase II, randomized trial which was recently presented at the 2020 ASCO Virtual Annual Meeting. This trial randomized 102 patients with resectable pancreatic adenocarcinoma to perioperative chemotherapy (12 weeks of neoadjuvant therapy followed by surgical resection followed 12 weeks of adjuvant therapy) with either mFOLFIRINOX or gemcitabine/nab-paclitaxel (33). Patients had to have a confirmed diagnosis of pancreatic ductal adenocarcinoma, ECOG performance status of $0-1$, resectable disease and 
could not have had any prior treatment to be eligible for this study. The primary end point was 2-year OS. Approximately equal numbers of patients in each treatment arm started (96\%) and completed (84\% vs. $85 \%)$ preoperative chemotherapy, underwent surgical resection ( $73 \%$ vs. $70 \%$ ) and started post-operative chemotherapy (56\% vs. 55\%). More patients completed the full course of post-operative mFOLFIRINOX (49\%) compared to those that received gemcitabine/nab-paclitaxel (40\%). Neither regimen met the pre-specified threshold of $58 \%$ OS at 2 -years. The mOS for mFOLFIRINOX was 22.4 months with 2 -year OS of $43.1 \%$ compared to mOS of 23.6 months and 2 -year OS of $46.9 \%$ for gemcitabine/nab-paclitaxel. Equal numbers of patients achieved R0 resection (85\%) in both treatment arms. Interestingly, the rates of complete or major pathologic responses and node negative resections were higher among those that received gemcitabine/nabpaclitaxel ( $42 \%$ and $45 \%)$ compared to mFOLFIRINOX (25\% and $40 \%$ ). DFS also favored the gemcitabine/nabpaclitaxel arm (14.2 months) over the mFOLFIRINOX arm (10.9 months). This study concluded that perioperative mFOLFIRINOX and gemcitabine/nab-paclitaxel have similar efficacy in this setting and have acceptable safety and resectability rates.

These data are compelling for the use of neoadjuvant therapy in resectable pancreas adenocarcinoma and more time and data from ongoing studies will be needed to determine which approach is optimal, neoadjuvant $v$ s. adjuvant, especially in light of the encouraging results of the PRODIGE-24-PA6 trial. The NCCN now includes a neoadjuvant treatment approach among the recommended treatment options for the management of resectable pancreatic cancer. Recommended regimens in the neoadjuvant setting include either FOLFIRINOX/ mFOLFIRINOX or gemcitabine/nab-paclitaxel with or without radiation therapy prior to surgery (3). For a clinically fit patient with ECOG performance status of 0-1, FOLFIRINOX/mFOLFIRINOX is recommended. In those patients that are not candidates for FOLFIRINOX, gemcitabine/nab-paclitaxel is recommended. It is highly recommended that patients be seen and evaluated at high-volume centers to determine resectability and recommendations for treatment. Further, it is highly encouraged for these patients to be enrolled to a clinical trial in this setting if eligible and willing to participate.

\section{Ongoing neoadjuvant clinical trials for resectable pancreas adenocarcinoma}

There are several key phase II and III randomized clinical trials which are ongoing to further evaluate the efficacy of neoadjuvant treatment in resectable pancreatic adenocarcinoma. See Table 3. The NEPAFOX trial (NCT02172976) is a German phase II/III clinical trial for patients with resectable or borderline resectable pancreas adenocarcinoma and patients will be randomized to either surgery followed by 6 cycles of adjuvant gemcitabine or to neoadjuvant FOLFIRINOX (4-6 cycles) followed by surgical resection and 4-6 cycles of adjuvant FOLFIRINOX. The NEONAX trial (NCT02047513) is a German phase II clinical trial for patients with resectable pancreas adenocarcinoma which will randomize patients to either upfront surgical resection followed by 6 cycles of adjuvant gemcitabine/nab-paclitaxel or to 2 cycles of neoadjuvant gemcitabine/nab-paclitaxel followed by surgical resection and 4 cycles of adjuvant gemcitabine. The PANACHE-01 trial (NCT02959879) is a French phase II study for patients with resectable pancreas adenocarcinoma which will randomize patients to either 4 cycles of neoadjuvant FOLFOX followed by surgical resection and 8 cycles of adjuvant FOLFOX, or 4 cycles of neoadjuvant FOLFIRINOX followed by surgical resection and 8 cycles of adjuvant FOLFIRINOX, or to upfront surgical resection followed by 12 cycles of standard adjuvant chemotherapy. The NorPACT-1 trial (NCT02919787) is a multicenter European phase II/III clinical trial for patients with resectable head of the pancreas adenocarcinoma and will randomize patients to either upfront surgical resection followed by adjuvant FOLFIRINOX or to 4 cycles of neoadjuvant FOLFIRINOX followed by surgical resection and adjuvant FOLFIRINOX.

\section{Borderline resectable pancreas adenocarcinoma}

Borderline resectable pancreas adenocarcinoma is defined as disease with abutment but lack of encasement of either the SMA or CA and/or reconstructable involvement of the SMV or PV or removable retroperitoneal structures (3). The impact of local vascular involvement has significant impact on the ability to achieve an R0 resection, and therefore has long-term impact on outcomes. 
Table 3 Ongoing randomized phase II or III clinical trials of neoadjuvant treatment in pancreas adenocarcinoma

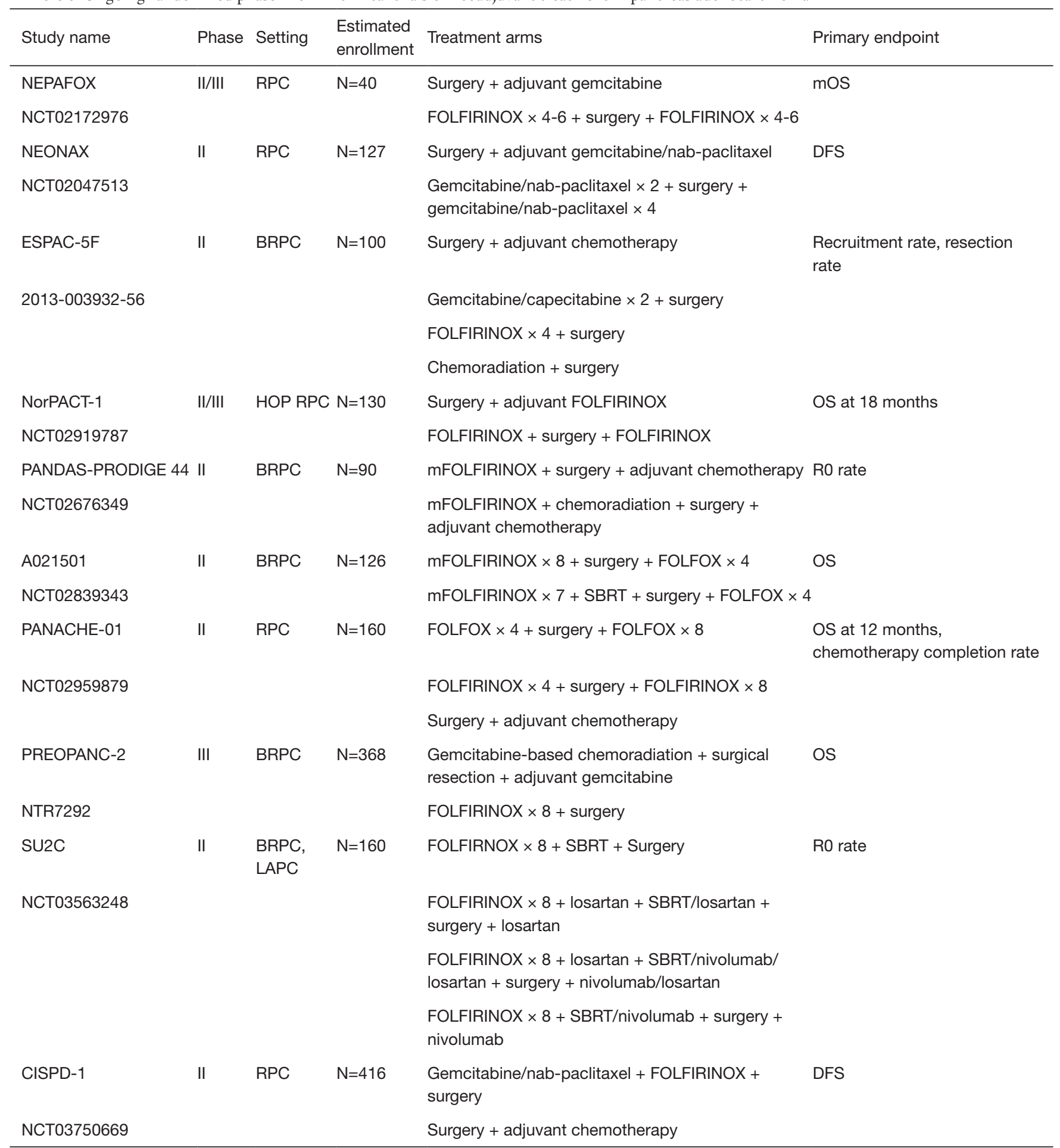

BRPC, borderline resectable pancreas cancer; DFS, disease free survival; FOLFIRINOX, fluorouracil, leucovorin, irinotecan, oxaliplatin; FOLFOX, fluorouracil, leucovorin, oxaliplatin; HOP, head of pancreas; LAPC, locally advanced pancreas cancer; mFOLFIRINOX, modified FOLFIRINOX; OS, overall survival; RPC, resectable pancreas cancer; SBRT, stereotactic body radiation treatment. 
A single-arm, single-institution, phase II, clinical trial of neoadjuvant FOLFIRINOX in patients with newly diagnosed, treatment naïve, borderline resectable pancreas adenocarcinoma was conducted with the primary endpoint of R0 resection rate (21). Forty-eight patients were included and received 8 cycles of neoadjuvant FOLFIRINOX followed by restaging imaging, and in those who had resolution of vascular involvement, short-course proton chemoradiation with capecitabine was administered. $\mathrm{R} 0$ resection was achieved in 31 of 48 eligible patients, with an $\mathrm{R} 0$ resection rate of $97 \%(\mathrm{n}=31 / 32)$ among those who underwent surgical resection. Median PFS among all eligible patients was 14.7 months with mOS of 37.7 months (95\% CI, 19.4-not reached). Outcomes were far superior among those patients that underwent surgical resection, compared to the ITT population, with a mPFS of 48.6 months and mOS not reached. A retrospective cohort review of borderline resectable pancreas adenocarcinoma patients treated at MD Anderson Cancer Center included 160 patients that were treated with neoadjuvant chemotherapy, chemoradiation or both (34). Among the cohort, $66(41 \%)$ underwent surgical resection. The mOS of the 160 patients was 18 months. However, there was a significant increase in mOS among those that underwent surgical resection (40 months) compared to those who did not undergo surgical resection (13 months; $\mathrm{P}<0.001)$. A retrospective cohort review of 120 patients with either borderline resectable or locally advanced pancreas adenocarcinoma treated with either neoadjuvant FOLFIRINOX or gemcitabine/nab-paclitaxel at the University of Colorado Hospital showed a similar rate of surgical resection in those that received neoadjuvant FOLFIRINOX (66.3\%) (20). Median PFS was 19.5 months and mOS was 27.4 months among this patient population.

A meta-analysis which included 24 studies (8 prospective and 16 retrospective) which included a total of 313 patients with borderline resectable pancreatic adenocarcinoma treated with neoadjuvant FOLFIRINOX was recently published (25). Among the included studies, the median number of administered cycles of FOLFIRINOX ranged from 4-9. The resection rate was $67.8 \%$ (95\% CI, 60.1$74.6 \%)$ and the R0 resection rate was $83.9 \%$ (95\% CI, $76.8-89.1 \%)$. Patient-level survival data was obtained from 20 of the studies included in analysis, consisting of 283 borderline resectable pancreatic adenocarcinoma patients, with mOS of 22.2 months (95\% CI, 18.8-25.6 months) and mPFS of 18.0 months (95\% CI, 14.5-21.5 months).
The NCCN recommends a neoadjuvant treatment approach for patients with borderline resectable disease, with either FOLFIRINOX/mFOLFIRINOX or gemcitabine/nab-paclitaxel with or without radiation therapy prior to surgery. While the majority of the completed neoadjuvant clinical trials have included only resectable pancreas adenocarcinoma, there are a number of ongoing large clinical trials which include borderline resectable patients (Table 3) which will hopefully further elucidate the role of neoadjuvant treatment in this setting and optimal sequencing of treatment.

\section{Ongoing neoadjuvant clinical trials for borderline resectable pancreas adenocarcinoma}

There are several ongoing randomized clinical trials to further evaluate the efficacy of neoadjuvant treatment in borderline resectable pancreas adenocarcinoma (Tables 3,4). The A021501 trial (NCT02839343) is a randomized phase II ALLIANCE trial for patients with biopsy proven borderline resectable pancreatic adenocarcinoma and will randomize patients to either 8 cycles of neoadjuvant mFOLFIRINOX followed by surgical resection and 4 cycles of adjuvant FOLFOX6 or 7 cycles of neoadjuvant mFOLFIRINOX followed by stereotactic body radiation therapy (SBRT) (33-40 Gy in 5 fractions) followed by surgical resection and 4 cycles of adjuvant FOLFOX6. The PREOPANC-2 trial (NTR7292) is a European randomized phase III study for patients with borderline resectable pancreas adenocarcinoma and will randomize patients to either neoadjuvant gemcitabine-based chemoradiation followed by surgical resection and adjuvant gemcitabine or 8 cycles of neoadjuvant FOLFIRINOX followed by surgical resection. The SU2C trial (NCT03563248) is a U.S. phase II randomized clinical trial for patients with borderline resectable or locally advanced pancreas adenocarcinoma and randomizes patients to one of four treatment arms, neoadjuvant FOLFIRINOX $\times 8$ cycles followed by SBRT and surgical resection, neoadjuvant FOLFIRINOX $\times$ 8 cycles plus losartan followed by SBRT plus losartan followed by surgical resection and 6 months of losartan, neoadjuvant FOLFIRINOX $\times 8$ cycles plus losartan followed by SBRT plus losartan and nivolumab followed by surgical resection and 6 months of losartan and nivolumab or neoadjuvant FOLFIRINOX $\times 8$ cycles followed by SBRT plus nivolumab followed by surgical resection and 6 months of nivolumab. 
Table 4 Select ongoing clinical trials in locally advanced pancreas adenocarcinoma

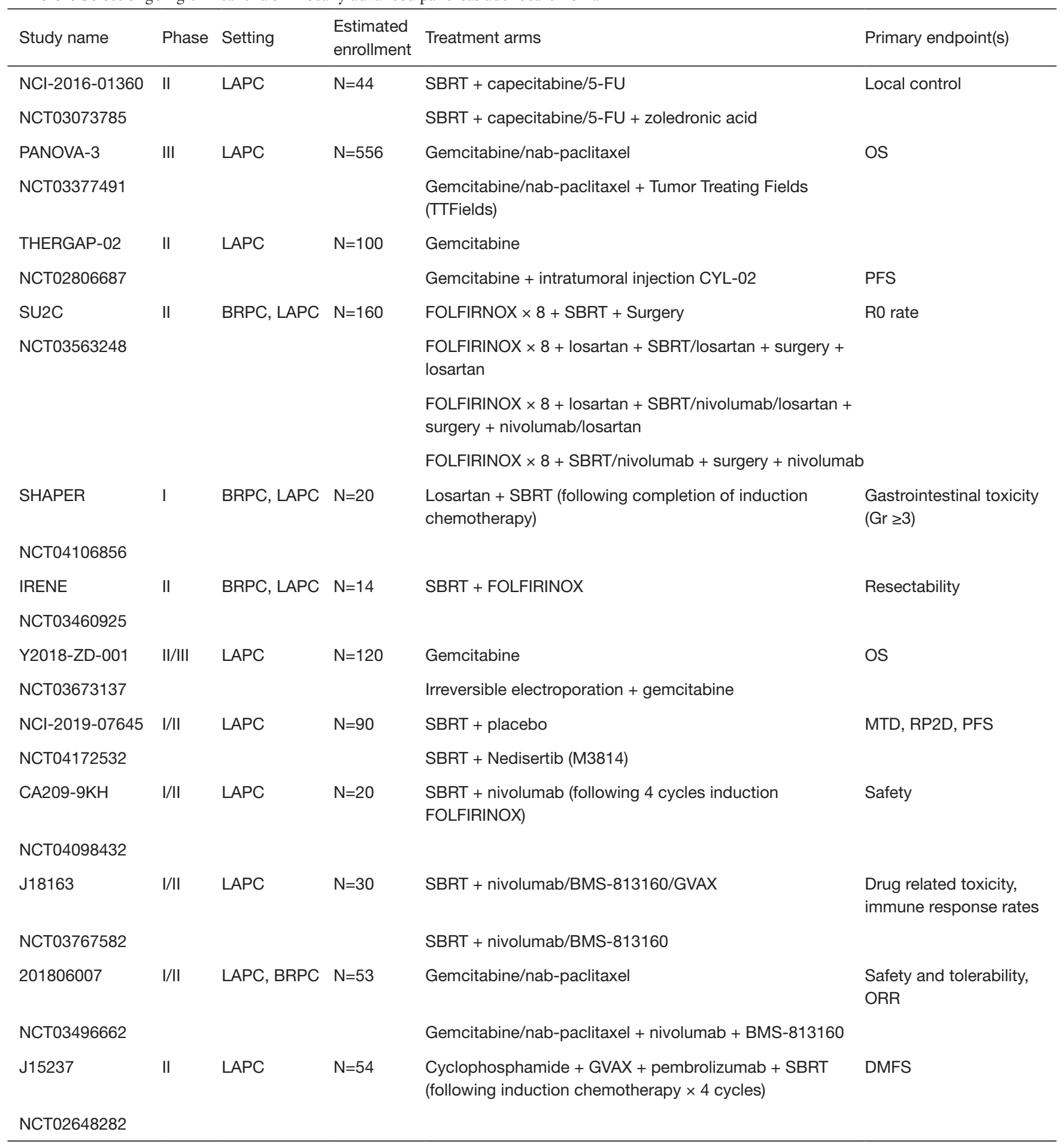

BRPC, borderline resectable pancreas cancer; DMFS, distant metastasis free survival; FOLFIRINOX, fluorouracil, leucovorin, irinotecan, oxaliplatin; Gr, grade; LAPC, locally advanced pancreas cancer; MTD, maximum tolerated dose; ORR, objective response rate; OS, overall survival; PFS, progression free survival; RP2D, recommended phase II dose; RPC, resectable pancreas cancer; SBRT, stereotactic body radiation treatment. 


\section{Locally advanced pancreas adenocarcinoma}

Approximately $30 \%$ of patients diagnosed with pancreatic adenocarcinoma present with locally advanced disease (35), which is defined as unresectable due to extensive local vascular involvement in the absence of metastases (3). These patients have primarily been treated with an induction approach, similar to that for borderline resectable patients, in the hopes of conversion to resectability following neoadjuvant treatment.

Several studies in locally advanced pancreas cancer patients comparing chemotherapy alone to chemoradiation have yielded conflicting findings, some showing a survival benefit with the addition of radiation $(36,37)$, while others have either not shown benefit or have shown harm $(38,39)$.

The LAP07 trial was an international, open-label, randomized phase III study which randomized 442 patients with locally advanced pancreas adenocarcinoma to induction chemotherapy with either gemcitabine or gemcitabine plus erlotinib (100 mg once daily) (39). Patients with stable disease or better after 4 months of induction treatment were then randomized to either two months of the same chemotherapy or chemoradiation with capecitabine (54 Gy). Four hundred and forty-two patients underwent first randomization, with 269 undergoing second randomization. This study was stopped early due to meeting early stopping boundaries for futility with no significant differences in survival between the chemotherapy and chemoradiation arms, with mOS 16.5 months for chemotherapy alone vs. 15.2 months for induction chemotherapy followed by chemoradiation (HR 1.03; 95\% CI, 0.79-1.34; $\mathrm{P}=0.83$ ). While this was a negative study, $4 \%(n=18)$ of patients were able to undergo curative intent surgical resection following induction chemotherapy. Six patients underwent resection after the first randomization and were excluded from the study, and twelve after the second randomization (6\% after chemotherapy alone, $3 \%$ after induction chemotherapy and chemoradiation). Of those who underwent resection, 11 had an R0 resection. For this subset of patients, mOS from the date of first randomization was 30.9 months (95\% CI, 12.3-not reached).

The NEOLAP trial was a German multicenter, openlabel, randomized, two arm, phase II trial which enrolled patients with biopsy proven, treatment naïve, unresectable locally advanced pancreas adenocarcinoma and randomized them to treatment with induction chemotherapy (40). Patients were initially treated with two cycles of induction gemcitabine/nab-paclitaxel and those without evidence of progressive disease or unacceptable toxicity were randomized 1:1 to either two additional cycles of gemcitabine/nab-paclitaxel or four cycles of FOLFIRINOX. Patients that had at least stable disease after completion of induction chemotherapy underwent surgical exploration to determine resectability. One hundred and thirty patients were allocated (64 to gemcitabine/nab-paclitaxel, 66 to FOLFIRINOX) and surgical exploration was performed in approximately $63 \%$ of patients in either treatment arm. Surgical conversion rate was the primary endpoint of this study and was $30.6 \%$ in the gemcitabine/nab-paclitaxel arm and $45 \%$ in the FOLFIRINOX arm $(\mathrm{P}=0.135)$. At median follow-up of 12.9 months, the mOS was 17.2 months in the gemcitabine/nab-paclitaxel arm compared to 22.5 months in the FOLFIRINOX arm (HR 0.73; 95\% CI, 0.42-1.28; $\mathrm{P}=0.268)$. Among the ITT population, surgical conversion was associated with significant improvement in survival (27.4 vs. 14.2 months; $\mathrm{P}=0.0035$ ).

It is recommended that patients with locally advanced disease be evaluated at a high-volume center with a multidisciplinary team approach. These patients should be treated with neoadjuvant therapy, with either induction FOLFIRINOX/mFOLFIRINOX or gemcitabine/nabpaclitaxel or enrollment on a clinical trial. Pending clinical response and lack of development of metastatic disease, consideration of chemoradiation or SBRT is recommended. Pending clinical response and disease stability, subsequent exploration or resection is recommended if feasible to do so.

\section{Ongoing neoadjuvant clinical trials for locally advanced pancreas adenocarcinoma}

There are several ongoing randomized clinical trials to further evaluate the efficacy of neoadjuvant treatment in locally advanced pancreatic adenocarcinoma (Table 4). The SU2C trial (NCT03563248) is a U.S. phase II randomized clinical trial for patients with borderline resectable or locally advanced pancreas adenocarcinoma and randomizes patients to one of four treatment arms, neoadjuvant FOLFIRINOX $\times 8$ cycles followed by SBRT and surgical resection, neoadjuvant FOLFIRINOX $\times$ 8 cycles plus losartan followed by SBRT plus losartan followed by surgical resection and 6 months of losartan, neoadjuvant FOLFIRINOX $\times 8$ cycles plus losartan followed by SBRT plus losartan and nivolumab followed by surgical resection and 6 months of losartan and nivolumab or neoadjuvant FOLFIRINOX $\times 8$ cycles followed by 
SBRT plus nivolumab followed by surgical resection and 6 months of nivolumab. There are several early phase studies investigating new agents in combination with chemotherapy or radiation and some novel treatment modalities to determine efficacy in this setting.

\section{Conclusions}

There have been considerable advances in the treatment of localized pancreatic adenocarcinoma over the last several years, with significant improvement in outcomes with multimodality treatment. However, despite these efforts, the long term outcomes for patients remain poor and therefore a dire need for new treatments and paradigms remains. Neoadjuvant therapy may improve outcomes and we await the emerging data from the pending and planned clinical trials to help further clarify and optimize therapeutic modalities to best serve patients with this disease.

\section{Acknowledgments}

Funding: None.

\section{Footnote}

Provenance and Peer Review: This article was commissioned by the Guest Editor (Elena Rangelova) for the series "Surgery for Locally Advanced Pancreatic Cancer" published in Fournal of Gastrointestinal Oncology. The article has undergone external peer review.

Conflicts of Interest: All authors have completed the ICMJE uniform disclosure form (available at http://dx.doi. org/10.21037/jgo-20-250). The series "Surgery for Locally Advanced Pancreatic Cancer" was commissioned by the editorial office without any funding or sponsorship. The authors have no other conflicts of interest to declare.

Ethical Statement: The authors are accountable for all aspects of the work in ensuring that questions related to the accuracy or integrity of any part of the work are appropriately investigated and resolved.

Open Access Statement: This is an Open Access article distributed in accordance with the Creative Commons Attribution-NonCommercial-NoDerivs 4.0 International License (CC BY-NC-ND 4.0), which permits the noncommercial replication and distribution of the article with the strict proviso that no changes or edits are made and the original work is properly cited (including links to both the formal publication through the relevant DOI and the license). See: https://creativecommons.org/licenses/by-nc-nd/4.0/.

\section{References}

1. Siegel RL, Miller KD, Jemal A. Cancer statistics, 2020. CA Cancer J Clin 2020;70:7-30.

2. Li D, Xie K, Wolff R, et al. Pancreatic cancer. Lancet 2004;363:1049-57.

3. NCCN Guidelines Version 1. 2020 Pancreatic Adenocarcinoma. Available online: https://www.nccn.org/ professionals/physician_gls/pdf/pancreatic.pdf

4. Kalser MH, Ellenberg SS. Pancreatic cancer. Adjuvant combined radiation and chemotherapy following curative resection. Arch Surg 1985;120:899-903.

5. Neoptolemos JP, Dunn JA, Stocken DD, et al. Adjuvant chemoradiotherapy and chemotherapy in resectable pancreatic cancer: a randomised controlled trial. Lancet 2001;358:1576-85.

6. Neoptolemos JP, Stocken DD, Friess H, et al. A randomized trial of chemoradiotherapy and chemotherapy after resection of pancreatic cancer. N Engl J Med 2004;350:1200-10.

7. Neoptolemos JP, Stocken DD, Tudur Smith C, et al. Adjuvant 5-fluorouracil and folinic acid vs observation for pancreatic cancer: composite data from the ESPAC-1 and -3(v1) trials. Br J Cancer 2009;100:246-50.

8. Oettle H, Neuhaus P, Hochhaus A, et al. Adjuvant chemotherapy with gemcitabine and long-term outcomes among patients with resected pancreatic cancer: the CONKO-001 randomized trial. JAMA 2013;310:1473-81.

9. Douglass HO. Further Evidence of Effective Adjuvant Combined Radiation and Chemotherapy Following Curative Resection of Pancreatic-Cancer. Cancer 1987;59:2006-10.

10. Neoptolemos JP, Stocken DD, Bassi C, et al. Adjuvant chemotherapy with fluorouracil plus folinic acid vs gemcitabine following pancreatic cancer resection: a randomized controlled trial. JAMA 2010;304:1073-81.

11. Uesaka K, Boku N, Fukutomi A, et al. Adjuvant chemotherapy of S-1 versus gemcitabine for resected pancreatic cancer: a phase 3, open-label, randomised, noninferiority trial (JASPAC 01). Lancet 2016;388:248-57.

12. Neoptolemos JP, Palmer DH, Ghaneh P, et al. Comparison of adjuvant gemcitabine and capecitabine with gemcitabine monotherapy in patients with resected pancreatic cancer 
(ESPAC-4): a multicentre, open-label, randomised, phase 3 trial. Lancet 2017;389:1011-24.

13. Sinn M, Bahra M, Liersch T, et al. CONKO-005: Adjuvant Chemotherapy With Gemcitabine Plus Erlotinib Versus Gemcitabine Alone in Patients After R0 Resection of Pancreatic Cancer: A Multicenter Randomized Phase III Trial. J Clin Oncol 2017;35:3330-7.

14. Conroy T, Hammel P, Hebbar M, et al. FOLFIRINOX or Gemcitabine as Adjuvant Therapy for Pancreatic Cancer. N Engl J Med 2018;379:2395-406.

15. Tempero MA, Reni M, Riess H, et al. APACT: phase III, multicenter, international, open-label, randomized trial of adjuvant nab-paclitaxel plus gemcitabine (nab-P/ $\mathrm{G})$ vs gemcitabine $(\mathrm{G})$ for surgically resected pancreatic adenocarcinoma. J Clin Oncol 2019;37:4000.

16. Reni M, Riess H, O'Reilly EM, et al. Phase III APACT trial of adjuvant nab-paclitaxel plus gemcitabine (nab-P + Gem) versus gemcitabine (Gem) alone for patients with resected pancreatic cancer (PC): Outcomes by geographic region. J Clin Oncol 2020;38:4515.

17. Crane CH, Ben-Josef E, Small W Jr. Chemotherapy for pancreatic cancer. N Engl J Med 2004;350:2713-5.

18. Morris SL, Beasley M, Leslie M. Chemotherapy for pancreatic cancer. N Engl J Med 2004;350:2713-5.

19. Bydder S, Spry N. Chemotherapy for pancreatic cancer. N Engl J Med 2004;350:2713-5.

20. Chapman BC, Gleisner A, Rigg D, et al. Perioperative and Survival Outcomes Following Neoadjuvant FOLFIRINOX versus Gemcitabine Abraxane in Patients with Pancreatic Adenocarcinoma. JOP 2018;19:75-85.

21. Murphy JE, Wo JY, Ryan DP, et al. Total Neoadjuvant Therapy With FOLFIRINOX Followed by Individualized Chemoradiotherapy for Borderline Resectable Pancreatic Adenocarcinoma: A Phase 2 Clinical Trial. JAMA Oncol 2018;4:963-9.

22. O'Reilly EM, Perelshteyn A, Jarnagin WR, et al. A single-arm, nonrandomized phase II trial of neoadjuvant gemcitabine and oxaliplatin in patients with resectable pancreas adenocarcinoma. Ann Surg 2014;260:142-8.

23. Gillen S, Schuster T, Meyer Zum Buschenfelde C, et al. Preoperative/neoadjuvant therapy in pancreatic cancer: a systematic review and meta-analysis of response and resection percentages. PLoS Med 2010;7:e1000267.

24. Dhir M, Malhotra GK, Sohal DPS, et al. Neoadjuvant treatment of pancreatic adenocarcinoma: a systematic review and meta-analysis of 5520 patients. World J Surg Oncol 2017;15:183.

25. Janssen QP, Buettner S, Suker M, et al. Neoadjuvant
FOLFIRINOX in Patients With Borderline Resectable Pancreatic Cancer: A Systematic Review and Patient-Level Meta-Analysis. J Natl Cancer Inst 2019;111:782-94.

26. Ye M, Zhang Q, Chen Y, et al. Neoadjuvant chemotherapy for primary resectable pancreatic cancer: a systematic review and meta-analysis. HPB (Oxford) 2020;22:821-32.

27. Merkow RP, Bilimoria KY, Tomlinson JS, et al.

Postoperative complications reduce adjuvant chemotherapy use in resectable pancreatic cancer. Ann Surg 2014;260:372-7.

28. Reni M, Balzano G, Zanon S, et al. Safety and efficacy of preoperative or postoperative chemotherapy for resectable pancreatic adenocarcinoma (PACT-15): a randomised, open-label, phase 2-3 trial. Lancet Gastroenterol Hepatol 2018;3:413-23.

29. Jang JY, Han Y, Lee H, et al. Oncological Benefits of Neoadjuvant Chemoradiation With Gemcitabine Versus Upfront Surgery in Patients With Borderline Resectable Pancreatic Cancer: A Prospective, Randomized, Openlabel, Multicenter Phase 2/3 Trial. Ann Surg 2018;268:21522.

30. Unno M, Motoi F, Matsuyama Y, et al. Randomized phase II/III trial of neoadjuvant chemotherapy with gemcitabine and S-1 versus upfront surgery for resectable pancreatic cancer (Prep-02/JSAP-05). J Clin Oncol 2019;37:189.

31. Satoi S, Unno M, Motoi F, et al. The effect of neoadjuvant chemotherapy with gemcitabine and S-1 for resectable pancreatic cancer (randomized phase II/III trial; Prep-02/ JSAP-05). J Clin Oncol 2019;37:4126.

32. Versteijne E, Suker M, Groothuis K, et al. Preoperative Chemoradiotherapy Versus Immediate Surgery for Resectable and Borderline Resectable Pancreatic Cancer: Results of the Dutch Randomized Phase III PREOPANC Trial. J Clin Oncol 2020;38:1763-73.

33. Sohal D, Duong MT, Ahmad SA, et al. SWOG S1505: Results of perioperative chemotherapy (peri-op CTx) with mfolfirinox versus gemcitabine/nab-paclitaxel (Gem/nabP) for resectable pancreatic ductal adenocarcinoma (PDA). J Clin Oncol 2020;38:4504.

34. Katz MH, Pisters PW, Evans DB, et al. Borderline resectable pancreatic cancer: the importance of this emerging stage of disease. J Am Coll Surg 2008;206:833-46.

35. Hidalgo M. Pancreatic cancer. N Engl J Med 2010;362:1605-17.

36. Huguet F, Andre T, Hammel P, et al. Impact of chemoradiotherapy after disease control with chemotherapy in locally advanced pancreatic adenocarcinoma in GERCOR phase II and III studies. J 
Clin Oncol 2007;25:326-31.

37. Loehrer Sr PJ, Powell ME, Cardenes HR, et al. A randomized phase III study of gemcitabine in combination with radiation therapy versus gemcitabine alone in patients with localized, unresectable pancreatic cancer: E4201. J Clin Oncol 2008;26:4506.

38. Chauffert B, Mornex F, Bonnetain F, et al. Phase III trial comparing intensive induction chemoradiotherapy (60 Gy, infusional 5-FU and intermittent cisplatin) followed by maintenance gemcitabine with gemcitabine alone for locally advanced unresectable pancreatic cancer. Definitive results of the 2000-01 FFCD/SFRO study. Ann Oncol

Cite this article as: Leal AD, Messersmith WA, Lieu CH. Neoadjuvant treatment of localized pancreatic adenocarcinoma. J Gastrointest Oncol 2021;12(5):2461-2474. doi: 10.21037/jgo-20250
2008;19:1592-9.

39. Hammel P, Huguet F, van Laethem JL, et al. Effect of Chemoradiotherapy vs Chemotherapy on Survival in Patients With Locally Advanced Pancreatic Cancer Controlled After 4 Months of Gemcitabine With or Without Erlotinib: The LAP07 Randomized Clinical Trial. JAMA 2016;315:1844-53.

40. Kunzmann V, Algül H, Goekkurt E, et al. Conversion rate in locally advanced pancreatic cancer (LAPC) after nabpaclitaxel/gemcitabine- or FOLFIRINOX-based induction chemotherapy (NEOLAP): Final results of a multicenter randomised phase II AIO trial. Ann Oncol 2019;30:V253. 